\title{
Structural assessment of myocardial infarction scars and left ventricular function with cardiac magnetic resonance imaging in patients at high risk of sudden cardiac death
}

\author{
Neuschl $V^{1}$, Berecova $Z^{2}$, Madaric $\mathrm{J}^{3}$, Simkova $\mathrm{J}^{1}$, Glezlova $\mathrm{A}^{1}$, Hatala $\mathrm{R}^{3}$ \\ Institute of Diagnostic Imaging, MRI sro, Trnava, Slovakia. neuschl.vlado.vn@gmail.com
}

\begin{abstract}
OBJECTIVES: Results of qualitative and quantitative analyses of scars and LV (left ventricle) function acquired by means of cardiac magnetic resonance (CMR) were correlated with a subsequent occurrence of malignant ventricular arrhythmias in patients at high risk of sudden cardiac death (SCD).

METHODS: We have prospectively followed 47 patients (mean age $60 \pm 11$ years) who were hospitalized for an implantable cardioverter-defibrillator (ICD) implantation to prevent SCD. All post-MI patients had severe residual LV dysfunction (LVEF 33 $\pm 14 \%$ ). Patients were examined with CMR. Based on CMR analysis, we evaluated the basic functional parameters of LV as well as mass, volume, transmurality and heterogeneity of the post-MI scar. RESULTS: The patients with malignant arrhythmias were characterized by smaller LV end-diastolic diameters (LVED $192 \pm 79$ vs $254 \pm 47 \mathrm{~mm}, \mathrm{p}=0.003$ ) and end-systolic diameters (LVES $131 \pm 80$ vs $181 \pm 45 \mathrm{~mm}, \mathrm{p}$ $=0.01)$. As for the other observed functional and morphological CMR parameters, no significant differences between the two groups were detected.

CONCLUSION: These results indicate that post-MI patients with severe residual left ventricular dysfunction and dilatation are in the long term characterized by a lower incidence of malignant arrhythmias compared to the patients with less dilated LV with a comparably severe LV dysfunction (Tab. 2, Fig. 3, Ref. 26). Text in PDF www.elis.sk. KEY WORDS: myocardial infarction, cardiac magnetic resonance imaging, ventricular tachyarrhythmias, sudden cardiac death, implantable cardioverter defibrillator.
\end{abstract}

\begin{abstract}
Abbreviations: CMR - cardiac magnetic resonance, $\mathrm{CV}$ - cardiovascular, ICD - implantable cardioverter defibrillator, IZD institute of diagnostic imaging, MI - myocardial infarction, LGE - late gadolinium enhancement, LVEF- left ventricular ejection fraction, LVED - left ventricular end-diastole, LVES - left ventricular end-systole, MRI - magnetic resonance imaging, SCD sudden cardiac death, SD - standard deviation.
\end{abstract}

\section{Introduction}

Sudden cardiac death (SCD) affects annually more than 1 million people in Europe and North America and it continues to be a serious medical and social problem of the developed world (1). In Slovakia, up to 8000 out-of-hospital cardiac arrests occur annually (2). The most common cause of sudden death is malignant ventricular tachyarrhythmia - ventricular tachycardia and/or ventricular

${ }^{1}$ Institute of Diagnostic Imaging, MRI sro, Trnava, Slovakia, ${ }^{2}$ Department of Radiology, St Michal Hospital, Bratislava, Slovakia, and ${ }^{3}$ Department of Arrhythmias and Cardiac Pacing, Academic Division of Cardiology and Angiology, Medical Faculty, Slovak Medical University and National Cardiovascular Institute - NUSCH Bratislava, Slovakia

Address for correspondence: V. Neuschl, MD, Institute of Diagnostic Imaging, MRI sro, Starohajska 2, SK-917 01 Trnava, Slovakia. Phone: +421332933230, Fax: +421332933232 fibrillation. The occurrence of malignant ventricular arrhythmias is closely linked to fibrotic morphological substrate, typically an infarction scar (3). Currently the only reliable strategy to prevent arrhythmic SCD is the implantation of a cardioverter - defibrillator (ICD). Most ICDs are implanted in patients at high risk of arrhythmic death as a primary preventative therapy. In majority of patients, the high risk of SCD is determined by the presence of chronic heart failure either of ischemic or non-ischemic etiology. The typical malignant arrhythmogenic substrate is represented by old myocardial infarction scarring.

The key role in identifying these high-risk patients is played by the presence of left ventricular systolic dysfunction expressed by the reduction in left ventricular ejection fraction (LVEF). Thus, a post-MI patient with LVEF $<35 \%$ (in absence of any other disease with a severe quad vitam prognosis) is according to all major international guidelines assigned to class I A for a primary preventative implantation of ICD (2015 ESC recommendation for the management of patients with ventricular arrhythmia and prevention of sudden cardiac death; 4).

Although such simplified risk stratification for SCD has an acceptable negative predictive value, its positive prediction value is rather limited. Current research in this area of cardiology is focused on new approaches to SCD risk stratification techniques in order to improve the accuracy of their predictive values (5). The 
259-264

new predictors can by classified as belonging to one of three categories as follows:

Complex computerized analysis of surface ECG signals with techniques for assessing the heart rate variability, Fourier spectral analysis in time and frequency domains, T-wave alternans, signal averaging, late ventricular potentials, etc.

Humoral biomarkers (e.g. natriuretic peptides, myocardial damage markers and other)

Imaging of proarrhythmogenic structural myocardial changes predisposing to malignant ventricular arrhythmias (echocardiography, magnetic resonance).

The aim of this study was to analyze several MRI-derived qualitative and quantitative parameters of LV myocardial structure and function in post-MI patients and to correlate CMR findings with the occurrence of death or malignant ventricular arrhythmias detected by ICD implanted shortly after the CMR examination.

\section{Patients and methods}

The observed cohort consisted of patients hospitalized in our specialized tertiary care institution and presented as potential candidates for ICD implantation in both primary and secondary forms of prevention. All patients had a significant coronary heart disease with the history of MI with residual severe LV dysfunction (EF $\leq 40 \%$ ). The total number of analyzed patients is 47 ; their mean age was $60 \pm 11$ years. The basic demographic characteristics of the cohort are listed in Table 1. During the observation period 2010 - 2017, five patients died suddenly and seven died of refractory congestive heart failure. Mortality data were obtained from statistical offices of the Slovak Republic (Bratislava and Trnava).

All analyzed patients were divided into two groups:

1. The group of patients with malignant arrhythmias ( $n=17$; Group

A) comprised three subgroups:

- Patients after ICD implantation in primary prevention with appropriate shocks,

- Patients after ICD implantation in secondary prevention (i.e. after spontaneous VT and/or aborted sudden death),

- Patients without ICD with sudden CV death.

2. The group of patients without malignant arrhythmias $(n=30$; Group B) comprised two subgroups:

- Patients with implanted ICD in primary prevention but without appropriate shocks,

- Surviving patients without ICD.

Two patients underwent surgical revascularization during the follow-up period and one patient underwent a successful heart transplantation.

\section{MR screening}

All investigations were performed at the magnetic resonance premises of a specialized imaging centre (IZD Inc., Trnava, Slovakia) equiped with CMR 1.5 T Avanto MRI scanner by Siemens, Germany. Investigations were supervised by a trained radiologist and individually evaluated by a radiologist with CMR specialization.

After data acquisition, the protocol for LV function evaluation and myocardial infarction scar detection was applied as follows:
Tab. 1. Basic demographic and clinical characteristics of the observed cohort.

\begin{tabular}{lc}
\hline Total number of patients (n) & 47 \\
Age (years) & $60 \pm 11$ \\
M : F & $41: 6$ \\
LVEF (\%) & $33 \pm 14$ \\
Number of primary preventative ICD implantations & 26 \\
Number of ICD implantations in secondary prevention & 5 \\
Patients without ICD with sudden cardiac death & 5 \\
Patients with ICD and heart failure death & 7 \\
\hline
\end{tabular}

1. Morphological sequences to distinguish pathological infiltrates in the myocardium (adipose deposits and other),

2. Multi-segmental sequences (for evaluation of regional and global LV functions),

3. Myocardium perfusion (rest/rest),

4. Aortic and pulmonary artery flow,

5. Ischemic and non-ischemic LV myocardial scar detection in 2D and 3D IR sequences.

Volumetric data were obtained in 2-chamber view by standard steady-state /TRUFI/ sequences. The scans were $6 \mathrm{~mm}$ thick and covered the entire left ventricle from base to apex (Fig. 7). The used settings were as follows: PAT factor 2, FOV 340x340 mm, matrix 256x256, flip angle 80', TR 36 mm, TE $1.2 \mathrm{~ms}$. Gadobutrol, an extracellular macrocyclic non-ionic gadolinium contrast agent, was administered to all patients (Gadovist $1 \mathrm{mmol} / \mathrm{ml} /$ manufactured by Bayer AG, Germany).

\section{Acquired MRI data analysis}

LVEF, left ventricle volume and mass were examined by commercially available cardio software within the Siemens-Argus function (Fig. 1). LV segments were set automatically, according to the American Heart Association (AHA) model with 17 segments according to the main coronary artery supply. Key data such as LVEF, LVED and LVES volumes and mass values were calculated.

Scar analysis included three aspects of quantifying the percentage of LV myocardial scarring, namely amount of MI, converted scar mass, transmurality, and heterogeneity count. The evalua-

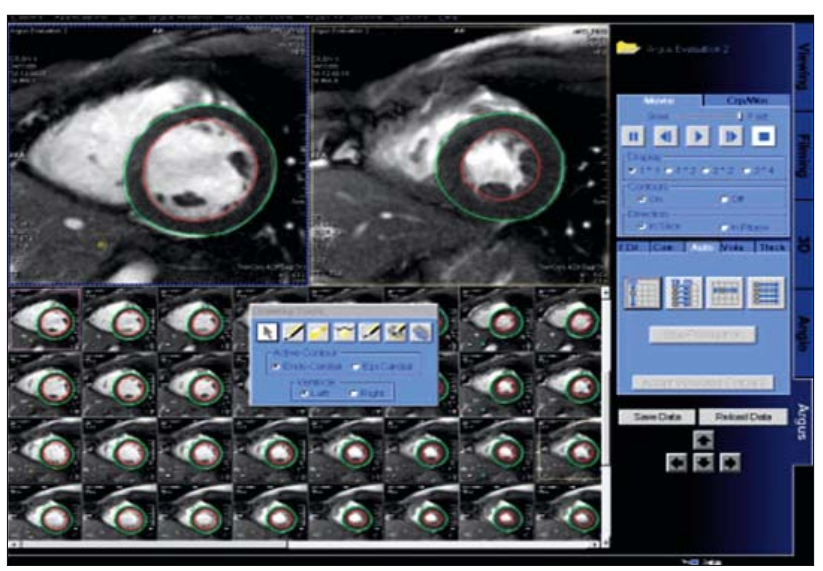

Fig. 1. CMR diagnostic console with Siemens Argus software to determine endo and epicardial contours of left ventricle in end-diastolic and end-systolic phases in short axis. (Figure from IZD archive in Trnava). 
tion was performed by means of Medis QMass diagnostic software (QMassMR version 8.1; Medis, Leiden, Netherlands.) The scarring was assessed by 2-SD technique /standard deviation/ (6).

The percentage of total scar size was calculated automatically with Q Mass program by summing all the hyperintense subendocardial to transmural myocardial zones of the left ventricle.

Scar heterogeneity was assessed visually when detecting intermediate scarring signal of the border zone.

Mass of the scar was calculated by multiplying the scar percentage with total LV mass in ED.

\section{Electrophysiological monitoring}

Thirty-one patients were implanted with an ICD, all as Class I indications according to ESC recommendations for the prevention of SCD (7). Twenty-six patients were implanted for primary prevention and 5 patients for secondary prevention ( $84 \%$ vs $26 \%$ ).

There were no changes in the health status of patients in the period between MR examination and ICD implantation. Patients were followed every 6 months and relevant data were provided by the Department of Arrhythmias and Cardiac Pacing of the National Cardiovascular Institute (NÚSCH a.s.) in Bratislava, Slovakia

\section{Statistical methods}

All continuous variables are presented as mean \pm SD. Normal distribution of the cohort division was tested by the KolmogorovSmirnov test. Mann-Whitney test (t-test) for comparing continuous data was used and Fisher exact test was used for the categorical variables. For all analyses, the value of $\mathrm{p}<0.05$ was considered statistically significant.

\section{Results}

Overall, 47 patients were prospectively followed after ICD implantation. All patients had coronary heart disease with a severe residual LV dysfunction (LVEF $33 \pm 14 \%$ ). Mean observation period was 2.7 years. Nine patients experienced adequate shocks (28 $\%$ of implanted patients). Seven ICD patients died non-suddenly.

When compared to patients from group B, patients with malignant arrhythmias (group A) were characterized by a lower LV end-diastolic diameter (LVED $=192 \pm 79$ vs $254 \pm 47 \mathrm{~mm}, \mathrm{p}=$ 0.003 ), as well as LV end-systolic diameter (LVES $=131 \pm 80$ vs $181 \pm 45 \mathrm{~mm}, \mathrm{p}=0.01$ ). No other significant differences were found in the observed functional and morphological MR parameters between the two groups (Tab. 2).

Tab. 2. MR parameters of the scar and left ventricle function.

\begin{tabular}{lccc}
\hline $\begin{array}{l}\text { CMR parameters of LV scar } \\
\text { and function }\end{array}$ & $\begin{array}{c}\mathrm{A} \\
\mathrm{n}=17\end{array}$ & $\begin{array}{c}\mathrm{B} \\
\mathrm{n}=30\end{array}$ & $\mathrm{p}$ \\
\hline Scar volume (\%) & $23 \pm 9$ & $25 \pm 8$ & 0.19 \\
LV mass (g) & $176 \pm 39$ & $185 \pm 38$ & 0.25 \\
Scar mass (g) & $41 \pm 16$ & $47 \pm 18$ & 0.15 \\
Scar transmurality (n) & 14 & 26 & 0.69 \\
Scar heterogeneity (n) & 12 & 24 & 0.49 \\
LVEF(\%) & $35 \pm 16$ & $30 \pm 8$ & 0.13 \\
EDV(ml) & $192 \pm 79$ & $254 \pm 47$ & 0.003 \\
ESV(ml) & $131 \pm 80$ & $181 \pm 45$ & 0.01 \\
\hline
\end{tabular}

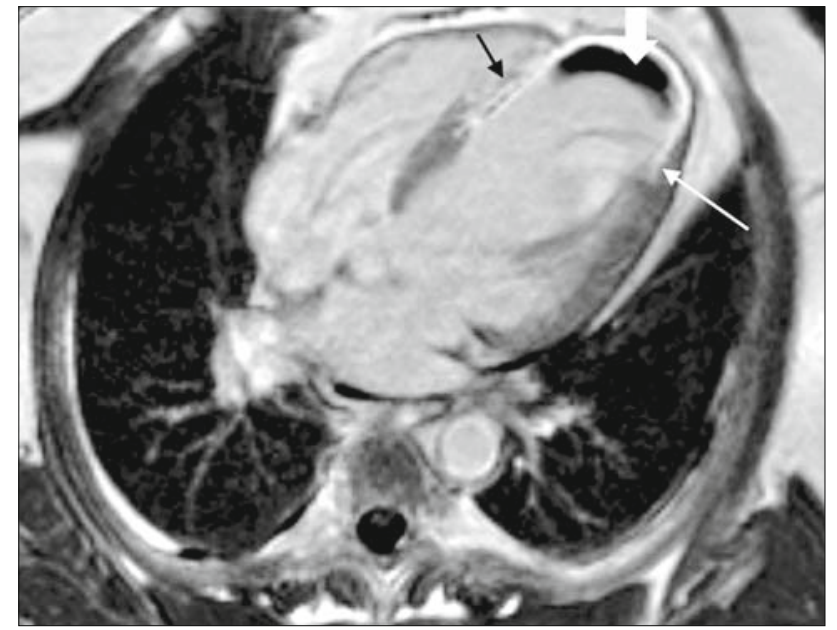

Fig. 2. Four-chamber LGE sequence projection in an individual patient. Thick arrow: non-viable myocardium and wall apex thrombus; long arrow: viable myocardium apico-lateral wall (scar size is 50 \% of the wall), short arrow: heterogeneous enhancement of the medial septum. (Figure from IZD archive in Trnava).

We compared LVEF assessed by MR to echocardiographic assessment performed at the same time as MR examinations. Based on echocardiographic assessment, mean LVEF in group A was $35 \pm 11 \%$, while in group B, it was $32 \pm 6 \%$. There were no significant differences between LVEF values assessed by MR and echocardiography (ECHO LVEF of group $\mathrm{A}+\mathrm{B} \mathrm{p}=0.11$ vs $\mathrm{CMR}$ LFEF of group $\mathrm{A}+\mathrm{B} \mathrm{p}=0.13$ ).

Furthermore, smaller post-MI scarring (7-12\% of LV mass) was detected in 3 patients, who clinically did not have MI.

Small LV mural thrombi (not identified by echocardiography) were detected in 4 out of 47 patients. The thrombus size in the long axis ranged from 7 to $12 \mathrm{~mm}$. All thrombi were localized apically, at the site of post-MI scarring (Fig. 2).

\section{Discussion}

In recent years, CMR research has upgraded this investigative method to a reference method of assessing left ventricular structure and function. Myocardial infarction lesions can be precisely visualized in all stages of their development. At the basis of CMR infarction lesion analysis is the technique of late enhancement of saturation after administering the contrast agent gadolinium (LGE). Fibrous tissue absorbs gadolinium, which results in higher signal strength. Bello et al. (8) hypothesized for the first time that LGECMR determined the size and morphology of the infarction deposit and might be a better predictor of ventricular tachycardia inducibility than traditional LVEF $<35 \%$. Patients with monomorphic ventricular tachycardia had greater infarction lesions compared to patients without inducible arrhythmia. CMR has a unique ability to visualize the character of peri-infarct zone, which, due to its heterogeneity, is considered to be part of the infarction deposit with the greatest arrhythmogenic potential $(9,10)$.

The main finding of our analysis is that post-MI patients with severe residual LV dysfunction and significantly dilated LV have 


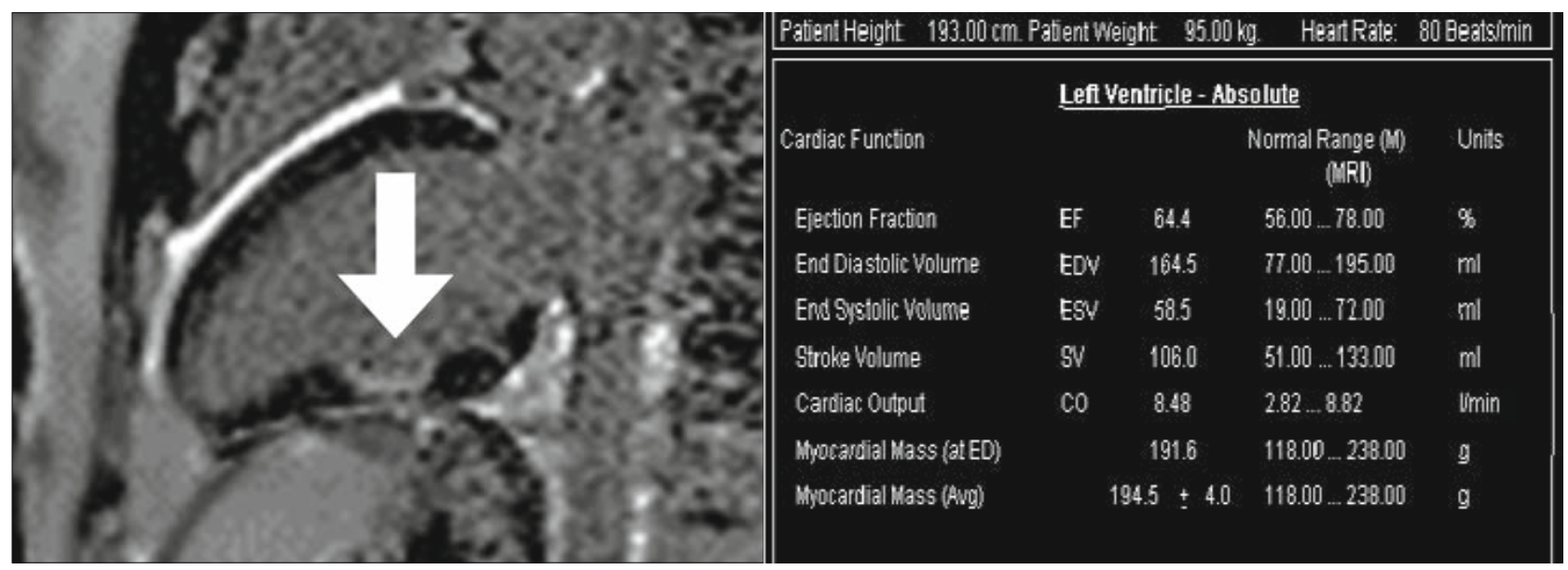

Fig. 3. MR scan of the left ventricle in long axis; patient with atypical chest pain, with nonspecific ECG and negative cardiac biomarkers. CMR finding of a small transmural scar of the lower wall. Scar volume: 5 \%; scar mass: 9 g; EF LV: 65 \% (patient not included in the study).

lower incidence of malignant arrhythmias compared to the patients with a comparable LV dysfunction but less LV dilatation. This observation offers a plausible support of the hypothesis, that a dysfunctional and massively dilated LV is prone to intractable pump failure and has lower arrhythmogenic potential compared to a less dilated, but dysfunctional ventricle. This finding might be the starting point of the hypothesis that the risk of sudden arrhythmic death is determined not only by the degree of LV dysfunction. The values of LV mass and LVEF, as well as those of other scar parameters were similar in both groups.

Our study did not confirm the expected differences of postinfarction scar parameters when comparing patients with malignant ventricular arrhythmias and sudden cardiac death to those without them. The underlying reasons for the fact that the differences in scar architecture between individual groups have not been detected are likely to be complex, both biologically and methodologically. As for the latter, a relatively small population with mixed ICD indications together with semiautomatic assessment of the scar may have contributed to our observations.

Statistically significant differences between the groups have been found in the exact volumetric sizes of end-diastolic and end-systolic LV volumes. We consider these data very reliable as CMR is a gold standard of non-invasive quantification of these measurements (11). Considerable changes in remodeling and left ventricular wall hypertrophy in group A may be expected. Additional parameters such as relative myocardial thickness, hypertrophy distribution and segmental kinetic evaluation of the wall were not evaluated in greater detail which might have increased the accuracy of observed findings.

Interestingly, there were some patients with no relevant clinical and/or ECG evidence of MI but with clear presence of smaller ischemic scars (4-12\%) (Fig. 3). According to some authors, they have a prognostic significance in CV mortality $(12,13)$. Autopsy findings reveal that $\mathrm{MI}$ can be considered one of the most frequently overlooked clinical diagnoses $(14,15)$. These non-identified MIs may be too small to be detected with ECG when MI takes place without typical chest pain symptoms (16). Non-identified MIs detected by MR occurred in a group of patients older than 70 years with a significant risk for adverse CV events (17). Similar findings were observed also in a sub-study of ICELAND-MI trial of AGES Reykjavik study (18) which compared patients with MI not obvious from ECG but clearly visualized by MR. The mortality in the latter group was similar to that of patients with clinically diagnosed MI.

More recent data (19) point out the predictive significance of the size of LV myocardium scar of non-coronary etiology, such as in hypertrophic cardiomyopathy. Scars exceeding $>15 \%$ of LV are associated with an increased risk of sudden cardiac death. These patients might benefit from primary preventative ICD therapy.

A thrombus in a heart chambers has a predominantly hypointense signal in LGE sequences and is well discriminated from the perifocal hyperintense myocardial scar. Four patients in our cohort had apical thrombi at the LV wall not detected by echocardiography. For detecting endocavitary thrombi, the contrast CMR examination has the highest sensitivity and specificity $(88$ $\pm 9 \%$ and $99 \pm 2 \%$, respectively) compared to TTE ( $23 \pm 12 \%$ and $96 \pm 3.6 \%$, respectively) and TEE ( $40 \pm 14 \%$ and $96 \pm 3.6 \%$, respectively) (20). Low LVEF and transmural myocardial scar were independent risk factors in the development of intracardiac thrombus (21). During a 6-month follow-up in the study, the incidence of cerebrovascular accident was $5.6 \%$ in thrombus patients and $2.1 \%$ in those without thrombus.

The use of different types of gadolinium contrast agent also affects the technique of measuring the post-MI scar $(22,23)$. A macrocyclic non-ionic gadolinium contrast agent, gadobutrol, was used in our study, which is recommended by some researchers for improving the contrast between LV cavity and myocardial scar (24).

\section{Study limitations}

The limitations of the submitted study can be divided into two primary groups; limitations of the study design and limita- 
tions of CMR examination. The particular limiting factor of the study design is the low number of patients from both groups with a relatively short follow-up (2.7 years). Analyzing a patient with both primary and secondary preventative ICD indications is also a limiting factor, but was used also in most similarly focused studies. Furthermore, several possible parameters of LV geometry were not studied, e.g. RWT (relative wall thickness), etc.

Basic CMR limitations include the impossibility to examine patients with ferromagnetic implants, including impulse generators manufactured prior to the MRI-compatible era. Furthermore, there are limiting CMR data in some patients with irregular heart beat.

Non-standard scar definition in a semi-automatic 2SD measurement methodology is the reason underlying the potential overestimation of signal intensity of the entire scar (25). Finally, the reference methods of non-invasive evaluation of the total amount of myocardial scarring are inaccessible. Experimental studies using histopathological techniques of scarring measurement from biopsy did not determine the total amount and scar distribution (26).

\section{Conclusion}

CMR represents a new dimension in the quality of heart imaging. In the settings of health-care system in the Slovak Republic it is still a heavily underused method. This paper has the ambition to point out clinically useful applications of CMR imaging for practicing cardiologists. Owing to its accuracy, non-invasiveness and radiation safety, CMR is undoubtedly the imaging method of the future. Contrary to severe risks linked to any X-ray imaging method, no adverse effects of CMR, with or without contrast agents, have been reported.

Study results acquired mainly in the past decade form a solid basis for the hypothesis that quantitative and qualitative analyses of morphological aspects of infarction scar may become a highly desirable complementary parameter of identifying patients at high risk of SCD. Scar detection in CMR imaging has a unique spatial resolution with the ability to capture not only the overall scar architecture but also to characterize the fine tissue structure. Thus, we believe that CMR is not only a precise tool for evaluating regional and global LV function, but has also an important potential to refine risk stratification of malignant ventricular arrhythmias in post-MI patients.

Our findings summarized in the presented study are only a very modest contribution to this topic, mainly due to the fundamental limitation of a relatively small number of patients (even though the majority of publications are also referring to less than 100 patients). In the structural parameters of infarction scars, namely in their extent and transmurality, no differences were found between groups with and without malignant arrhythmias. However, the finding that patients with severe left ventricular dysfunction and concomitant significant dilatation profit less from ICD implantation may be a very useful complementary piece of information for a clinically meaningful decision in primary SCD prevention using ICD.

\section{References}

1. Mozaffarian D, Benjamin EJ, Go AS et al. Heart disease and stroke statistics - 2015 update: a report from the American Heart Association. Circulation 2015; 131: e29-322. doi: 10.1161/CIR.00000000000001.

2. Gräsner JT et al. EuReCa ONE-27 Nations, ONE Europe, ONE Registry: A prospective one month analysis of out-of-hospital cardiac arrest outcomes in 27 countries in Europe. Resuscitation 2016; 105: 188-195. doi: 10.1016/j.resuscitation.2016.06.004.

3. Bayés De Luna A, Coumel P, Leclercq JF. Ambulatory sudden cardiac death: mechanisms of production of fatal arrhythmia on the basis of data from 157 cases. Am Heart J 1989; 117: 151-159.

4. Priori SG, Blomström-Lundquist C, Mazzanti A et al. ESC Guidelines for the management of patients with ventricular arrhythmias and the prevention of sudden cardiac death: The Task Force for the Management of Patients with Ventricular Arrhythmias and the Prevention of Sudden Cardiac Death of the European Society of Cardiology (ESC). Eur Heart J 2015; 36 (41): 2793-867. doi: 10.1093/eurheartj/ehv316.

5. Dagres N, Hindricks G. Risk stratification after myocardial infarction: is left ventricular ejection fraction enough to prevent sudden cardiac death? Eur Heart J 2013; 34: 1964-1971.

6. Fernández-Armenta $\mathbf{J}$ et al. Use of myocardial scar characterization to predict ventricular arrhythmia in cardiac resynchronization therapy. Europace 2012; 14: 1578-1586. doi: 10.1093/europace/eus104.

7. Priori SG, Blomström-LundquistC, Mazzanti A et al. 2015 ESC Guidelines for the management of patients with ventricular arrhythmias and the prevention of sudden cardiac death: The Task Force for the Management of Patients with Ventricular Arrhythmias and the Prevention of Sudden Cardiac Death of the European Society of Cardiology (ESC). Eur Heart J 2015; 36 (41): 2793-2867. doi: 10.1093/eurheartj/ehv316.

8. Bello D, Fieno DS, Kim RJ et al. Infarct morphology identifies patients with substrate for sustained ventricular tachycardia. J Am Coll Cardiol 2005; 45: 1104-1108.

9. Schmidt A, Azevedo CF, Cheng A et al. Infarct tissue heterogeneity by magnetic resonance imaging identifies enhanced cardiac arrhythmia susceptibility in patients with left ventricular dysfunction. Circulation 2007; 115: 2006-2014.

10. Zeidan-Shwiri T, Yang Y, Lashevsky I et al. Magnetic resonance estimates of the extent and heterogeneity of scar tissue in ICD patients with ischemic cardiomyopathy predict ventricular arrhythmia. Heart Rhythm 2015; 12: 802-808. doi: 10.1016/j.hrthm.2015.01.007.

11. Alfakih K, Reid S, Jones T et al. Assessment of ventricular function and mass by cardiac magnetic resonance imaging. Eur Radiol 2004; 14: 1813-1822.

12. Brieger D, Eagle KA, Goodman SG et al. Acute coronary syndromes without chest pain, an underdiagnosed and undertreated high-risk group: insights from the Global Registry of Acute Coronary Events. Chest 2004; 126: $461-469$.

13. Schelbert EB, Cao JJ, Sigurdsson S et al. Prevalence and prognosis of unrecognized myocardial infarction determined by cardiac magnetic resonance in older adults. JAMA 2012; 308: 890-896. doi: 10.1001/2012. jama.11089.

14. Perkins GD, Mcauley DF, Davies S et al. Discrepancies between clinical and postmortem diagnoses in critically ill patients: an observational study. Crit Care 2003; 7: R129 -132. 


\section{9-264}

15. Spiliopoulou C, Papadodima S, Kotakidis $\mathbf{N}$ et al. Clinical diagnoses and autopsy findings: a retrospective analysis of 252 cases in Greece. Arch Pathol Lab Med 2005; 129: 210-214.

16. Brieger D, Eagle KA, Goodman SG et al. Acute coronary syndromes without chest pain, an underdiagnosed and undertreated high-risk group: insights from the Global Registry of Acute Coronary Events. Chest 2004; 126: 461-469.

17. Barbier C, Bjerner T et al. Myocardial Scars More Frequent Than Expected: Magnetic Resonance Imaging Detects Potential Risk Group. J Amer Coll Cardiol 2006; 48: 765-771.

18. Schelbert EB, Cao JJ, Sigurdsson S et al. Prevalence and prognosis of unrecognized myocardial infarction determined by cardiac magnetic resonance in older adults. JAMA 2012; 308: 890-896. doi: 10.1001/2012. jama.11089.

19. Rowin EJ, Maron MS. The Role of Cardiac MRI in the Diagnosis and Risk Stratification of Hypertrophic Cardiomyopathy. Arrhyth Electrophysiol Rev 2016; 5: 197-202. doi: 10.15420/aer.2016:13:3.

20. Srichai MB, Junor C, Rodriguez LL et al. Clinical imaging, and pathological characteristics of left ventricular thrombus: a comparison of contrast-enhanced magnetic resonance imaging, transthoracic echocardiography, and transesophageal echocardiography with surgical or pathological validation. Am Heart J 2006; 152 (1): 75-84.
21. Weinsaft JW, Kim HW, Shah DJ et al. Detection of left ventricular thrombus by delayed enhancement cardiovascular magnetic resonance prevalence and markers in patients with systolic dysfunction. J Am Coll Cardiol 2008; 52: 148-157.

22. Durmus T, Schilling R, Doeblin $\mathbf{P}$ et al. Gadobutrol for magnetic resonance imaging of chronic myocardial infarction: intraindividual comparison with gadopentetate dimeglumine. Invest Radiol 2012; 47: 183-188.

23. Wildgruber M, Stadlbauer T, Rasper M et al. Single-dose gadobutrol in comparison with single-dose gadobenate dimeglumine for magnetic resonance imaging of chronic myocardial infarction at $3 \mathrm{~T}$. Invest Radiol 2014; 49: 728-734.

24. Wagner M, Schilling R, Doeblin P et al. Macrocyclic contrast agents for magnetic resonance imaging of chronic myocardial infarction: intraindividual comparison of gadobutrol and gadoterate meglumine. Eur Radiol 2013; 23: 108-114.

25. Flett AS, Hasleton $\mathbf{J}$ et al. Evaluation of techniques for the quantification of myocardial scar of differing etiology using cardiac magnetic resonance. JACC Cardiovasc Imaging 2011; 4: 150-156.

26. de Jong S, van Veen TAB, de Bakker JMT, van Rijen HVM. Monitoring cardiac fibrosis: a technical challenge. Neth Heart J 2012; 20 (1): 44-48. 